\title{
A Constructal Approach to Power Distribution Networks Design
}

\author{
A.M. Morega ${ }^{1}$, J.C. Ordonez ${ }^{2}$ and M. Morega ${ }^{1}$ \\ ${ }^{1}$ Department of Electrical Engineering \\ POLITEHNICA University of Bucharest \\ Splaiul Independentei nr. 313, 060042 Bucharest (Romania) \\ Phone:+0041 21 4029153, e-mail: amm@iem.pub.ro, ordonez@caps.fsu.edu, mihaela@iem.pub.ro \\ 2 Department of Mechanical Engineering, Florida State University, \\ Tallahassee, 032310, FL (USA) \\ e-mail: ordonez@caps.fsu.edu
}

\begin{abstract}
Superior, scalable grid topology that may provide for higher quality of service, survivorship capacity, and reconfiguration capability is a feature of modern grid architecture. The reported research is aimed at delivering a possible, constructal solution that is based on a minimumredundant, scalable, reconfigurable topology that helps increasing the grid immunity to faults. We assume that a tree network serves nodes of consumption that are evenly distributed throughout the territory. All nodes are equally important and network survivability means delivering electrical energy to as many consumers as possible. The models and loads estimation is based on the load momentum method.
\end{abstract}

\section{Key words}

Power Network, Reconfigurable, Scalable architecture, Constructal optimization, Models and estimation of loads.

\section{Introduction}

Power distribution networks depend on geography, technological epoch, economic policy and international relations. They serve large numbers of consumers and their role and performance may be analyzed in terms of different criteria, such as deliverability, cost, accessibility, reliability and complexity, to name some. By economic and political reasons, contemporary transmission networks are large and complex, spanning great distances and conveying power from many generators to many metropolitan areas located far away [1]-[4]. Recent blackout events were due to failures of transmission networks.

In this work we are interested in power network layouts that are more robust with respect to destructive events. Consequently, it is expected that more resources need to be allocated, and redundancy of sources and distribution lines is expected to occur. Therefore, a higher degree of topologic complexity should be accounted for. However, limiting factors such as costs are acting.

\section{An Integral Method for the Evaluation of Electric Power Networks}

Several design solutions to this multi-criteria problem are possibly available, each of them performing better than the others with respect to one or more criteria [5]. In evaluating their performance it is then important to use a method that reveals their underlying structure and performance, and this relates to loads $\left(I_{0}, P_{0}\right)$ and lines lengths [6]. This approach is common to many other classes of problems, such as water heating, telephone, data, and natural gas networks. In particular, for each of them the same design problem may be posed.

We use an integral approach [7]-[9], useful in the evaluation of the performance of a power distribution network design. This method accounts for the hierarchical, layered structure of tree shaped networks and for all the consumption nodes, links (lines) and loads carried by each line. By grouping all the lines that carry the same load, and by summing up the total lengths of the lines in each group, this analysis produces a load-length curve that represents the network - Fig. 1.

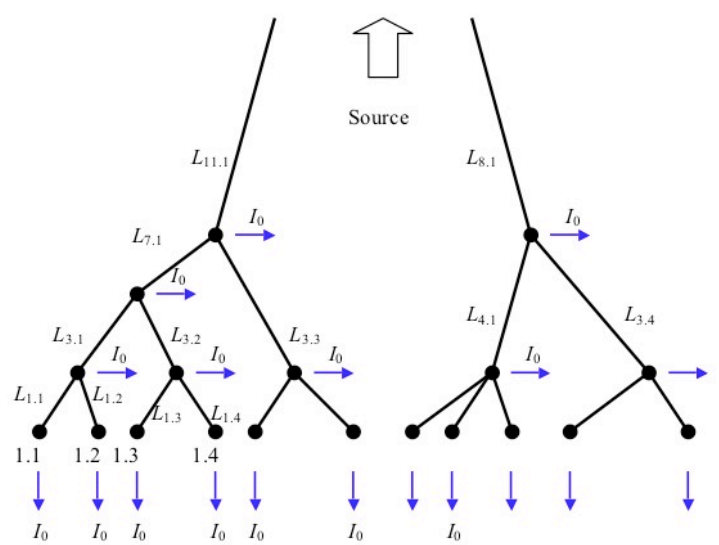

Fig. 1. Hierarchic representation of load momentum in a distribution network.

The (electrical) moment of the load at the consumption 
node is defined as the load of that node times the direct distance (the minimum spanning tree) from the node (sink) to the source - Fig. 2.

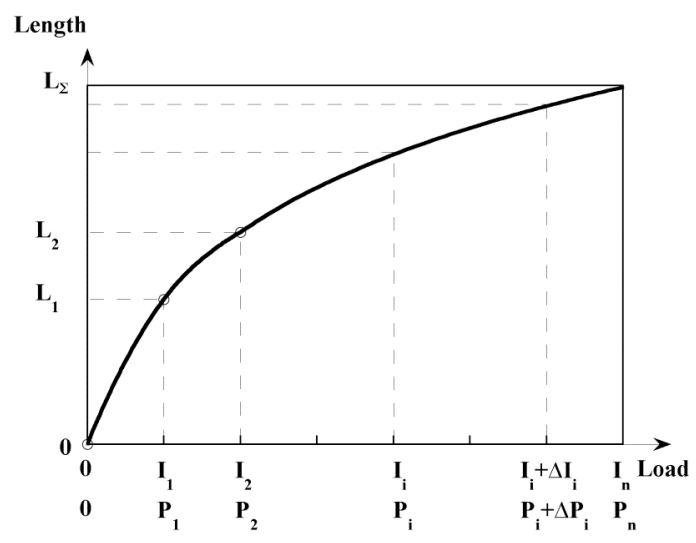

Fig. 2. Load length for currents and power.

The global load moment is then the sum of all the individual load moments. A consequence is that the global load moment is proportional to the area above the load-length curve of the network. This feature offers a direct mean of optimization - the area is to be minimized in order to minimize the discounted total cost of the network. This representation has several advantages:

- The load-length diagram shows-off the total length $\left(\Delta L_{i}\right)$ of the branches that must carry loads in a specific economic range, for instance, the range of $I_{i}+\Delta I_{i}$. This information is useful in sizing the network and estimating its total costs. In fact, the global moment is one of the most general and effective indicators of the electrical and economic performance of the distribution networks.

- The area above the curve is equal to the actual total electrical (load) moment at the source. Consequently, optimization may be related to the minimization of this area. More, the load-length diagram may be used to evaluate the load for a specific topology - e.g., for a post-failure recovery scenario - and to validate it against the location and availability of dormant sources, as seen next.

Several tree-shaped networks were reportedly investigated and compared on this integral basis. The load-length diagram may be used to size the network: it shows the total length of lines that have to carry a specific load, in a specific economic range. For instance, the group with a load in the range $I_{i} \ldots I_{i}+\Delta I_{i}$ covers a range of lengths $\Delta L_{i}$. Further more, the surface above the load-length curve is proportional to the total cost.

\section{A Constructal Design to Maximize Network Survivability under Dstructive Events}

The network model we propose comprises a source, located at the highest hierarchal level and a number of consumers, connected in a tree network. All nodes are equally important, such that the network survivability translates into delivering the electrical energy to as many consumers as possible. Special nodes, if any, should be served by special means.

The capacity of survivorship and recovery increases when the system possesses some redundancy to overcome failures, and this means sources and lines, able to take over areas that loose connectivity to their basic tree source. We assume that a second network functions in the same area and provides energy on a regular basis. To be capable of helping each other, the two networks are comparable in terms of size, regional coverage, and power flow, and the nodes of consumption are evenly distributed between the two networks. Although this model suggests two concurrent, competing networks, it turns out that when survivability is a matter of concern, two networks can perform better together than individually. The sources should be able then to support an acceptable level of overload: ideally, each of the sources should be able at sustaining both networks.

Redundancy of lines is also needed. The two networks may work completely independent, but to help each other supplementary lines and ancillary switching equipment are a must. Also, the regular lines should have a "passing through" capacity larger than that expected in nominal service, as they will occasionally carry overloads.

The internetworking strategy relies on the constructal theory [10]-[17], which is concerned with optimizing the access path from a volume (here, the territory where the nodes of consumption are located) to a point (here, the source), under given resources (the network), and subject to internal and external constraints (local restrictions, finite size area, ports of access to the territory).

Finally, we rely on the principle that the consumption nodes and the two spanning trees should be at close distance at all scales - as the circulatory system, or as counterflow systems.

\section{A. A Concurrent Flow System - A Related Boundary Value Problem}

In concurrent flow systems two trees are conveying current at all levels, form the elemental cell up to high order ensembles [9], [10]. As the object of our work is the concept for a reconfigurable network, connectivity (electrical current, power flow paths) is to be outlined first. To illustrate the constructal design, we consider a related electrokinetic boundary value problem: an area that sinks current uniformly and which has a low electrical conductivity $\left(\sigma_{0}<<\sigma_{p}\right)$ is fed through two high conductivity tree networks, which cover evenly the region of consumption (Fig. 3).

The amount of high conductivity material, $\sigma_{\mathrm{p}}$, the area of the cell (hence, the current demand) and the structure of the trees are given. The degree of freedom used in the constructal optimization problem (minimum highest voltage drop) is the cell aspect ratio, $H / L$. The optimization problem consists of finding the cell aspect 
ratio (shape) for which the transfer admittance, defined through the ratio between the largest voltage in the cell and the current input to the cell, is the smallest.

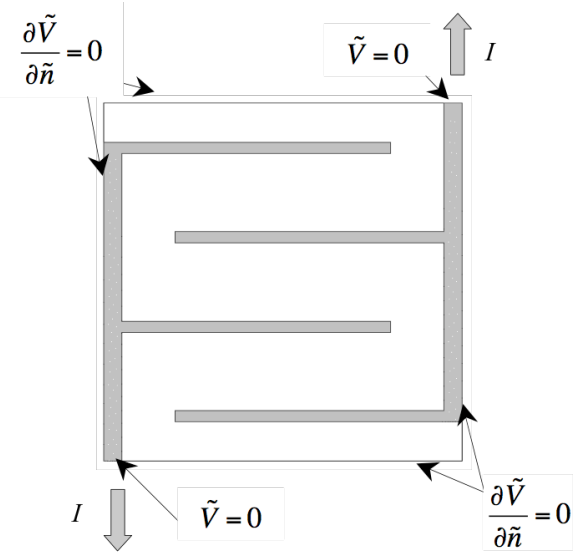

Fig. 3. The elemental cell of two concurrent tree networks. The boundary conditions.

The nondimensional form of the mathematical model of the steady state (DC) boundary value problem for the elemental cell is then

in the region that sinks current

$$
\frac{\partial^{2} \tilde{V}}{\partial \tilde{x}^{2}}+\frac{\partial^{2} \tilde{V}}{\partial \tilde{y}^{2}}-1=0,
$$

in the region that conveys the current

$$
\frac{\partial^{2} \tilde{V}}{\partial \tilde{x}^{2}}+\frac{\partial^{2} \tilde{V}}{\partial \tilde{y}^{2}}=0
$$

where $\tilde{V}=\left(V-V_{0}\right) / \Delta V, \quad \Delta V=w^{\prime \prime \prime} L^{2} / \sigma_{0}$. The length scale is $L$, the cell width, and $V_{0}$ is set to the ground value $(0 \mathrm{~V})$. We used COMSOL [18] multiphysics FEM software to solve the electric field problem. Accuracy tests showed that meshes of up to 145000 triangular, Lagrange cubic elements depending on the ensemble order, provide grid-independent numerical solutions.

Homogeneous Neumann conditions are assumed on all sides of the cell, except for the inlet ports (the boundaries that are orthogonal to the current carrying strips), where homogeneous Dirichlet conditions are set, Fig.3. In equations (1), (2) $V$ is the electrical potential and $w^{\prime \prime \prime}$ is the current sink, assumed uniform. The current density is related to the voltage by $\mathbf{J}_{p, 0}=-\sigma_{p, 0} \nabla V$ within the transport and sink regions; $\sigma_{p}$ and $\sigma_{0}$ are the electrical conductivities of the sink and transport regions.

Figure 4 presents the optimization sequence. The optimal design corresponds to the minimum voltage(s) (nondimensional). For comparison, the voltages are divided by the minimum values recorded for each sequence, respectively.

The optimization problem solved here is related to a boundary value problem, where the sink is continuously distributed throughout the domain.

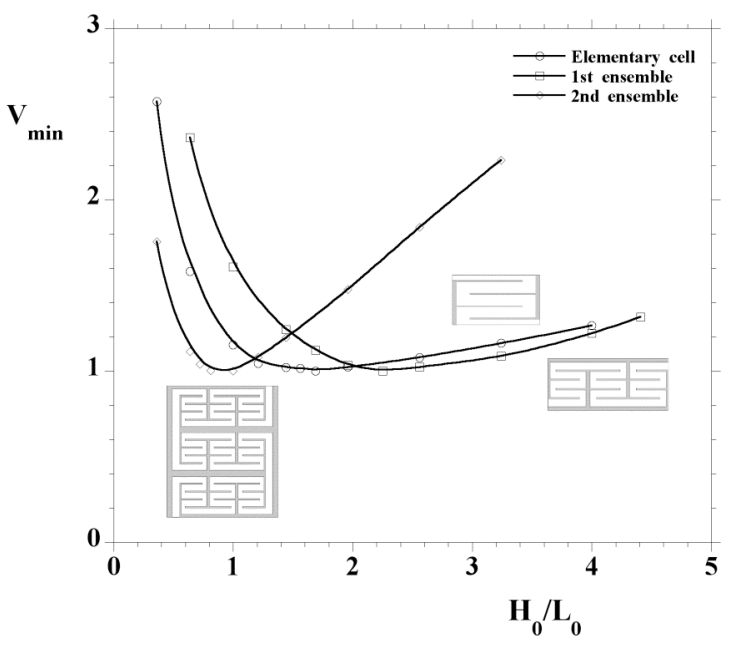

Fig. 4. Optimization sequence (non-dimensional).

Although different from the electrical network problem, where the sink is discrete (a set of nodes of consumption), directly connected to the grid, this problem provides an important message and a conceptual solution to the grid problem: the electrical network should bear the same major features - two concurrent trees, and the constructal design where the elemental cell generates higher order ensembles that posses, at each scale, the same properties.

\section{B. Concurrent Electrical Trees}

In electrical networks the sinks are discrete nodes of consumption rather than regions with continuous, uniform demand for current (power). In the electrical network layout that we present next, two trees are closely packed at each scale level, similar to the concurrent flow problem solved in the previous section. This design is called concurrent flow structure.

Next, we propose a reconfigurable network. Because topology and connectivity (electric current, power flow paths) are to be outlined first, the DC regime - although less general in electrical networking - provides for a simpler, satisfactory analysis of connectivity. Figure 5.a shows the elemental cell. The two trees, the electrical lines, the client consumption nodes and the local areas are marked in black and grey, respectively. Apparently, the networks share equal parts of the elemental cell. The nodes have the same load, $I_{0}, P_{0}$, and areas of dependence, $2 l_{0} \times 2 l_{0}$, (marked in grey). The first order ensemble is made of three elemental cells, as seen in Fig.5.b. Consumption nodes (dots) and switching nodes (dots and circles) outline the structure.

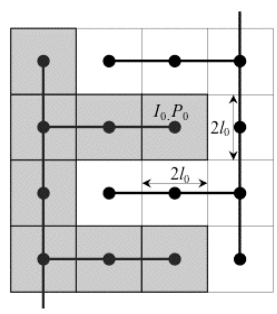

a. Elemental cell.

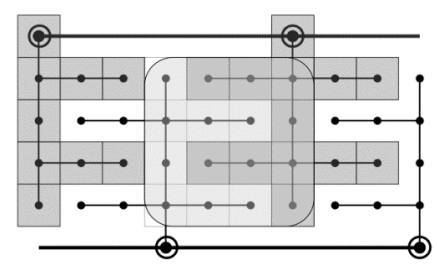

$\boldsymbol{b}$. The first order ensemble made of two first order elemental cells. 


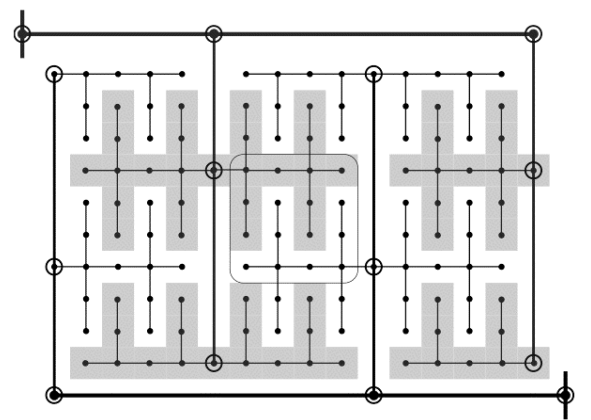

c. The second order ensemble made of three first order ensembles - an elemental cell is outlined.

Fig. 5. Concurrent flow networks.

Although the internetworking complexity is increasingly higher, the two trees are seen to coexist in a balanced share of their clients, without crisscrossing and loops. The topological scalability of this constructal structure is straightforward, already noticeable.

The second order ensemble results by merging three first order ensembles. The constructal morphologic growth goes on, in a time arrow that goes from the elemental cell to higher order ensembles, where each higher order ensemble is made of three ensembles of immediate lower level. The natural limit of this growth is the accepted size of the distribution network.

\section{The Electrical Load Moment}

Figure 6 presents two designs for the elemental cell. We assume that the elemental region is divided in identical square elemental patches, the centers of which are the consumption nodes, marked with full circles. Also, the consumption nodes drain the same current, $I_{0}$, or, equivalent, they request the same electrical power. The nodes marked with circled dots are for inter-connection (pass-through) purposes, and they do not represent consumption nodes. The line segments have the same length, $2 l_{0}$, which is the side of the elemental patch. These assumptions lead to simpler calculus in the models presented next, without loosing generality.

The consumption nodes in the grey network are numbered (1...8), and the non-dimensional load for each line segment is written aside, in parentheses - the actual values are divided by $I_{0}$, or $P_{0}$. The two lines line area equally loaded. The non-dimensional load moment (divided by $2 l_{0} I_{0}$ ) at node $(\mathbf{A})$ is shown too.

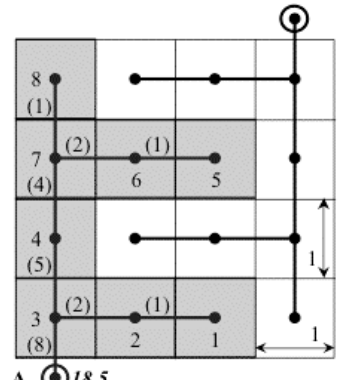

A 18.5 a. Design 1.

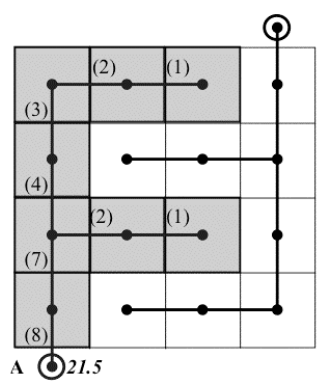

b. Design 2.

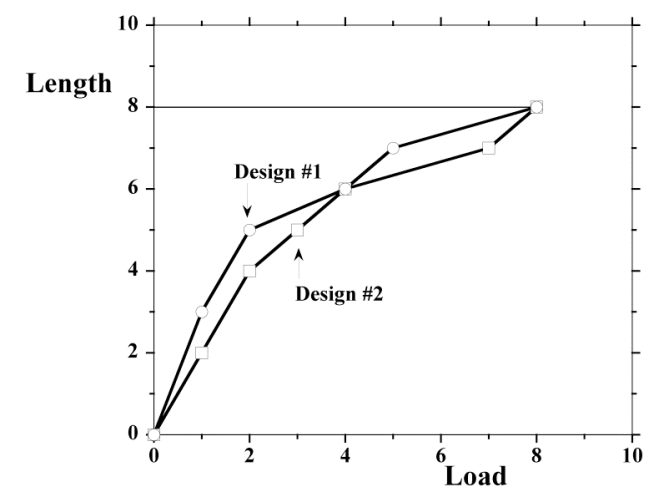

c. The load-length diagrams for the two candidate cells

Fig. 6 . The total momentum optimization.

Clearly, design 1 is superior as its total load momentum (the area above the curve) is smaller - 18.5 as compared to 21.5. The total load moment is also closely related to the discounted (present worth) total cost of the network, which must account for investment costs, maintenance costs and the costs of power and energy losses during the lifetime. It was shown that when the network is complex, sized economically and designed to serve consumers that cover the territory uniformly the discounted total cost is

$$
\mathrm{TCD}=4 l_{0} I_{0} C_{m} K_{m} .
$$

Here $C_{m}(\$ / \mathrm{A} \mathrm{km})$ is the specific cost of the load moment

$$
C_{m}=k_{I} E_{\Sigma} / j_{e c}=3 \rho k_{I} E_{\Sigma} C_{p w}^{\prime} .
$$

Here, $\rho$ is the conductor resistivity $\left(\Omega \mathrm{mm}^{2} / \mathrm{km}\right), k_{I}$ is the specific investment cost of the line $\left(\$ / \mathrm{mm}^{2} \mathrm{~km}\right)$, and $j_{e c}$ is the economic current density $\left(\mathrm{A} / \mathrm{mm}^{2}\right)$. The factor $E_{\Sigma}=$ $1+\alpha_{M}-\alpha_{B}$ accounts for the annual cost of maintaining the lines $\left(\alpha_{M}\right)$ and the book value of the assets at the end of the planned period $\left(\alpha_{B}\right)$. The factor $C_{p w}^{\prime}$ is the total cost of the demand and energy losses, expressed per unit of demand loss $(\$ / \mathrm{kW})[7]-[8]$.

The elemental load moment, $M_{0}=2 l_{0} I_{0}$ may also be viewed as $\mathrm{TCD}=\mathrm{TCD}_{0} K_{m}$, where $\mathrm{TCD}_{0}$ is the total cost associated with the element length $2 l_{0}$ and load $I_{0}$, hence $\mathrm{TCD}_{0}=4 l_{0} I_{0} C_{m}$. The carried load and the total load moment of the network are then proportional to the total cost, which adds value to the load momentum approach to sizing the network. Alternatively, TCD $=2 M C_{m}$, where $M$ is the total load moment and $M C_{m}$ is the cost of the moment. The total cost is then twice the cost of the total load moment. Since the surface above the length-load diagram is proportional to the total cost, competing network configurations may be evaluated and compared early, without having to estimate their total costs.

In what follows we use the load-length diagram to evaluate the performance of the alternative post-failure recovery topologies, define thus a basis for quantitative comparison and decide what layouts do more efficiently respond to functional and economical criteria.

Figure 7 reports the non-dimensional loads for the first order ensemble. The load moments at nodes $\mathbf{A}, \mathbf{F}$, are noted too. The grey and the black lines carry the same load and withstand the same moments. 


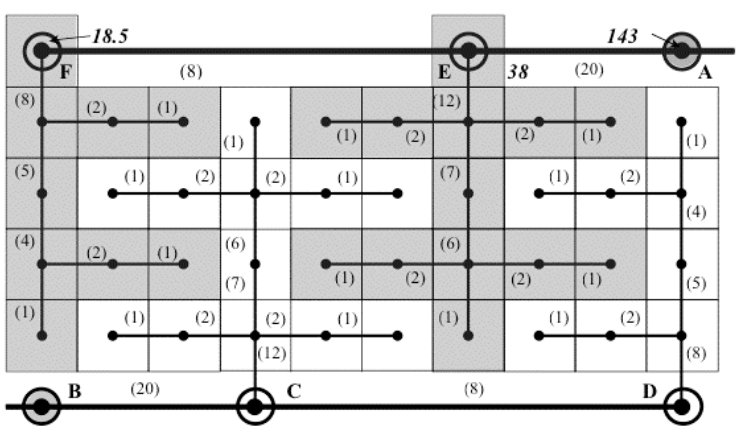

Fig. 7. The load moment for the first order ensemble concurrent tree networks.

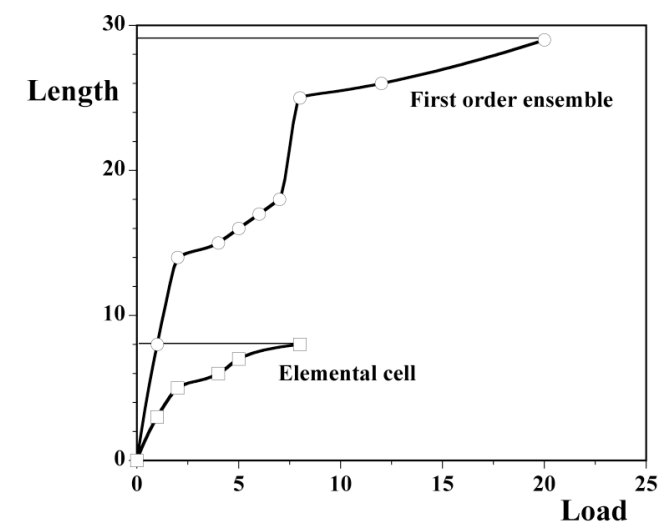

Fig. 8. Nominal load-length diagram - non-dimensional.

The total (non-dimensional) load moments for the elemental cell and the first order ensemble are equal to the areas above the curves (Fig. 8).

Similar information may be reported for the higher order ensembles. We restrict the analysis at the first order ensemble level. Its complexity is illustrative for higher order constructs while requesting moderate calculus.

\section{A Fault Recovery by Reconfiguration}

The concurrent flow design proposed in this study provides for several features such as topological scalability, redundancy, and reconfiguration capacity that are important for defining any successful post-failure recovery plan. These features are inherited from the constructal growth algorithm that generates the structure of the network.

Redundancy occurs here in two respects: (i) the number of trees that make the constructal network, at all levels of complexity - two instead of a single one; (ii) the specific topology permits more than one solution to recover form a failure. The two trees may belong either to a single phase (a mono-phased network), or to two distinct phases (the trees attached to different phases).

Additional redundancy concerning supplementary switching capacity, dormant sources, etc. - not addressed here - is sizable based on a trade-off analysis between the possible solutions provided by the constructal network, the relevance (importance) that some of the consumption nodes may have, and the pending costs.
There are many types of failures that may occur. However, in many situations the direct consequence of a failure (fault) is that the distribution network becomes an "island", disconnected from its source node(s). The constructal, concurrent flow design we propose may help solving this difficulty.

The fact that at any level of complexity two networks rather than a single one exist may provide for the needed redundancy to ensure alternative paths for power flow to the islandized region - either at a consumption node level or at a subnet level.

To exemplify this we present two possible failure scenarios that may occur by faults at the first ensemble level. The black network in Fig. 9 is supposedly under failure, such that part of it is islandized and has to be disconnected from its source.

There are many ways to connect the (black) island to the grey network, which is assumed fully operational. Evidently, the supplementary load that the grey network would have to carry in this situation, and the passthrough capacity of the supplementary line from the islandized subnet to the withstanding line are crucial in making a decision. Figure 9 shows a straightforward connection, via node $\mathbf{A}$ that would tie up the island without exceeding, hopefully, the load that the lowest level lines have to withstand. To implement it, nodes $\mathbf{C}$ and $\mathbf{D}$ - pass-through nodes - should be able to isolate the fault.

Figure 10 shows the non-dimensional length-load curve for the grey and black trees that result as compared to their nominal length-load curve.

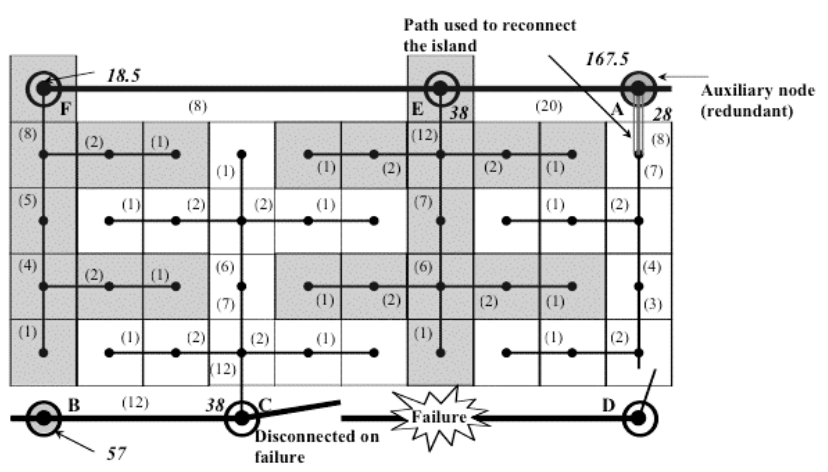

Fig. 9. An islandized subnet taken over by the second network. The numbers in parentheses are loads and those in italics are electrical (load) moments - non-dimensional.

The grey network load curve is shifted upwards indicating heavier working conditions, whereas the load curve of the still working part of the black network is shifted downwards, which indicates a lighter load. The non-dimensional total load moments are proportional to the areas above the curves.

Figure 10 shows the non-dimensional length-load curve for the grey and black trees that result as compared to their nominal length-load curve. 


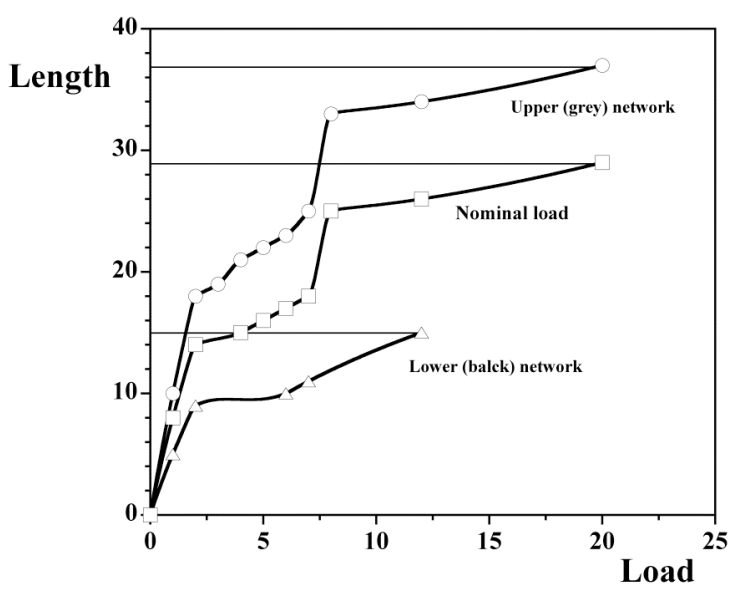

Fig. 10. The load-length diagrams for the recovery scenario shown in Fig. 9.

The reconfiguration prescribed by this scenario leads thus to an uneven distribution of loads between the two networks, and it implies the capability of node $\mathbf{A}$ to switch the islandized subnet to the blue network - in this example $\mathbf{A}$ is not a consumption node.

\section{Conclusion}

The first phase of a roadmap to the new generation grid is the architecture: the conceptual design, prototyping, and field-testing, and it concerns all grid levels: the backbone, the regional interconnections and the local distribution.

The fundamental problem that we had to answer in this study is the following: how to connect a collection of consumption nodes in a robust, reliable net that would provide for recovery capacity, flexibility in reconfiguration, with minimum redundancy, hence, costs.

The capacity of survivorship and recovery increases when the system possesses some redundancy to overcome failures. Therefore we think that, qualitatively, two trees are needed. However, the two-tree network may be reduced to a one-tree network. This extends our analysis' scope from two-sources networks to one-source networks, which in this approach become a subset of the two-sources networks class.

The constructal theory unifies engineered and natural flows that connect an infinite number of points (volume, area) to one or more discrete points (sources, sinks).

The networking problem solved is related to a field model, where the sink is continuously distributed throughout the domain. Although different from the electrical network problem, where the sink is discrete (a discrete set of nodes of consumption), directly connected to the grid, the field problem provides an important message and a solution to the network topology.

Apparently, only two tree networks may be combined in an interweaved, planar, non-intersecting scalable structure, and the proposed constructal design may be the single possible planar design in topological sense.
The AC model for the network provides a fairly accurate approximation of the steady state behavior of a power grid. DC model is vastly popular in the electric power literature, being the model of choice any time that many power flow computations are needed, or in the design phase when topology and connectivity are evaluated.

The length-load integral method, widely used in sizing large-scale networks is built on the DC model. It gives a concise picture of the electrical (moment) load (current or power) that a network supports, and the quantitative means to quantify it. By using it, post-failure recovery scenarios may be evaluated, for complex networks.

There are many types of failures that may occur, and an exhaustive analysis of this issue was beyond the scope of the current work. However, in many situations the consequence of a failure is that the distribution network becomes an "island", disconnected from its generation node(s). The constructal, concurrent flow design we propose may help solving this difficulty. The fact that $a t$ any level of complexity two network rather than a single one exist may provide for the needed redundancy to ensure alternative paths for power flow to the islandized subnet, and the examples we presented document reconfiguration strategies in post-failure scenarios.

Specific applications may be either the construction or the development of distribution networks in residential areas, camps, compounds, and also indoor networking with a certain degree of spatial regularity and power consumption request, and where lines, cables and bus bars may be posed according to the typical, two-tree concurrent network. The planar nature of the proposed network has important technological advantages here too. Multi-phase powered distribution areas may take advantage of the two-trees topology. Reconfiguration is then a matter of transferring the islandized subnet(s) from one phase (under failure) to the other (withstanding).

The proposed design may have potential in structuring networks with distributed generation too, and for developing robust local DC networks.

Concluding, the outcome of this trail are: a concept: the planar constructal topology made of two concurrent, interdigitated networks that is scalable, minimum redundant and robust that we propose; and a method to quantify the electrical load of a specific network configuration. Constructal topology provides alternative scenarios of recovery from failure, such that cost effective solutions may be identified.

\section{Acknowledgements}

A. Morega gratefully acknowledges the support provided through the research grants CEEX 05-D11-25/05.10.05, CNCSIS 358/2007. J.C. Ordonez acknowledges with gratitude the support from the Office of Naval Research through the ESRDC program and the U.S. Department of Energy, Office of Electricity Delivery and Energy Reliability, under Award no. DE-FG02-05CH11292. 


\section{References}

[1] Gonnen T., Electric power distribution systems engineering, New York, McGraw-Hill, 1986.

[2] Willis H., Power distribution planning reference book, New York, Marcel Dekker, 1997.

[3] IEEE recommended practice for electric power distribution for industrial plants (Red Book), New York, IEEE, 1994.

[4] Lakervi E., Holmes E.J., Electricity distribution network design, $2^{\text {nd }}$ Ed., London, Peter Peregrinus, 1995.

[5] McCalley J., Kumar R., Elia N., Ajjarapu, Vittal V., Liu H., Jin L., "Planning on reconfigurable power systems", in 2004 EPNES Workshop, Mayaguez, Puerto Rico, 12-14 July, 2004.

[6] Poeata A., Arie A., Crisan O, Eremia M., Alexandrescu M, Buta A, Transportul si distributia energiei electrice, Ed. Pedagogica, Bucuresti 1981.

[7] Arion V., Cojocari A. and Bejan, A., "Constructal tree shaped networks for the distribution of electrical power," Energ. Conversion Management, 44 (2003), pp. 867-891.

[8] Arion V., Cojocari A. and Bejan, A., "Integral measures of electric power distribution networks: load-length curves and line-network multipliers," Energ. Conversion Management, 44 (2003), pp. 1039-1051.

[9] Morega A.M., Ordonez J.C., "Constructal Design of Concurrent Power Distribution Networks", in COMSOL Conference, Boston, October 20-21,
Hyatt Regency, Cambridge, Boston, MA, 2006.

[10] Bejan A. Shape and Structure, from Engineering to Nature, Cambridge Univ. Press, 2000.

[11] Poirier H. Une theorie explique l'intelligence de la nature, in Science \& Vie, 1034 (2003) pp. 44-63.

[12] Vargas J.V.C., Ordóñez, J.C., Bejan, A. "Constructal Flow Structure for a PEM Fuel Cell," International Journal of Heat and Mass Transfer, 47 (2004), pp. 4177-4193.

[13] Ordóñez J.C., Bejan, A., Cherry, R.S., "Designed Porous Media: Optimally Nonuniform Flow Structures Connecting One Point With One Or More Points," International Journal of Thermal Sciences, 42 (2003), pp. 857-870.

[14] Morega A.M., Bejan A., "A constructal approach to the optimal design of photovoltaic cells," Int. J. Green Energy, 2, 3, pp. 233-242, 2005

[15] Negoias, A.P., Morega, A.M. and Ordonez, J.C.,'Heat transfer in spherical photovoltaic cells", in ATEE'06, Bucuresti, 24 Nov., 2006

[16] Morega, A.M., Ordonez, J.C., Negoias, P.A., Morega, M., and Hovsapian, R., "Optimal electrical design of spherical photovoltaic cells", COMSOL Conference, Congress Center of the Czech Technical University, Prague, October 26, 2006.

[17] Morega, A.M., Ordonez, J.C., Negoias, A.P. and Hovsapian R., "Spherical photovoltaic cells - A constructal approach to their optimization", OPTIM 2006, May 2006, Brasov, Romania.

[18] Comsol A.B., v.3.3a, Sweden, 2006, 2007. 